Doh Jeing Yong, MBBS, MRCS

Iskandar Hailani, MBBS, MS (ORL-HNS)'

Mohd Razif bin Mohamad Yunus, MBBS, MS (ORL-HNS)2

\section{'Department of Otorhinolaryngology}

Hospital Kuala Lumpur, Malaysia

2Department of Otorhinolaryngology

Universiti Kebangsaan Malaysia Medical Centre

Kuala Lumpur, Malaysia
Correspondence: Doh Jeing Yong, MBBS, MRCS

Department of Otorhinolaryngology,

Hospital Kuala Lumpur, Jalan Pahang, 50586

Kuala Lumpur, Malaysia

Phone: +60122719921

Fax: +60326916725

Email: drdjyong@gmail.com

Reprints will not be available from the authors.

The authors declared that this represents original materia that is not being considered for publication or has not been published or accepted for publication elsewhere, in full or in part, in print or electronic media; that the manuscript has been read and approved by all the authors, that the requirements for authorship have been met by each author, and that each author believes that the manuscript represents honest work.

Disclosures: The authors signed disclosures that there are no financial or other (including personal) relationships, intellectual passion, political or religious beliefs, and institutional affiliations that might lead to a conflict of interest.

\title{
Cervical Vagal Schwannoma
}

\begin{abstract}
Objectives: To present a case of cervical vagal schwannoma and describe our experience with the clinical presentation, surgical management and outcome of an elderly patient with this pathology.
\end{abstract}

\section{Methods:}

\author{
Design: Case Report \\ Setting: Tertiary Public Hospital \\ Patient: One
}

Results: A 65-year-old lady presented with a recently enlarging, pulsatile right sided neck mass that had been asymptomatic for 15 years. Contrast $\mathrm{CT}$ revealed a circumscribed non-enhancing heterogenous $4 \times 4 \times 7 \mathrm{~cm}$ mass splaying the right internal jugular vein and common carotid artery. A neurogenic tumour was considered, and the mass was excised from the vagus nerve with preservation of adjacent structures. Final histopathologic reading was schwannoma. However, the patient succumbed to complications following a second surgery for expanding hematoma.

Conclusion: Schwannomas are benign, slow growing tumours that arise from Schwann cells of the nerve sheath. Cervical schwannomas originating from the vagus nerve are rare but should be considered in patients presenting with solitary neck masses. Surgical extirpation is still the treatment of choice for nerve sheath tumours and recurrence is uncommon. Efforts should be made to preserve unaffected structures and patients should be counseled preoperatively on the possible high risk of morbidity especially in the elderly group where close follow up and aggressive rehabilitation should be instituted following surgery.

\section{Keywords: Cervical schwannoma, vagus nerve, neurogenic tumour; parapharyngeal space}

The head and neck region is a source of swellings of various types of pathology. Nonetheless, neurogenic tumours arising from these regions are uncommon in the adult population and are rare in the pediatric group. On the other hand, these tumours are commonly found arising from the parapharyngeal spaces. ${ }^{1}$ The reported sites of origin of neurogenic tumours are the cranial nerves $9-12$, the sympathetic chains, the cervical plexus and the brachial plexus. ${ }^{2-5}$ Cervical schwannomas are rare, slow-growing tumours of nerve sheath origin that may originate from any of these nerves. ${ }^{6}$ They are also commonly referred as neuromas and neurilemmomas. They are usually asymptomatic benign lesions and complete surgical resection is the treatment of choice. Malignant transformation is unusual. No gender predilection is noted and they are usually reported to occur in patients between 34 and 67 years of age. ${ }^{6}$ We report the case of a patient diagnosed with cervical schwannoma of vagal nerve origin, which is rare. 


\section{CASE REPORTS}

\section{CASE REPORT}

A 65-year-old lady with painless swelling on the right side of the neck for 15 years consulted at our centre for recent enlargement. She had no dysphagia, dyspnoea or other associated symptoms nor was there a past history of cervical trauma. Her past medical and surgical histories were unremarkable. On examination, the swelling was oval in shape and situated beneath the upper and middle third of the right sternocleidomastoid muscle. It measured $5 \times 3 \mathrm{~cm}$ with normal overlying skin. On palpation, the mass was firm in consistency, pulsatile and could be displaced from side to side but not up and down. No bruit was noted on auscultation. Regional lymph nodes were not enlarged. The intraoral, indirect laryngeal and post nasal mirror examinations were normal. No cranial nerve deficits or Horner's syndrome were noted. Flexible nasopharyngolaryngoscopy showed no significant findings. In view of the pulsatility of the neck mass, contrasted computed tomography scan (CT) of the neck was performed and revealed a well circumscribed non-enhancing heterogenous mass measuring $4 \times 4 \times 7 \mathrm{~cm}$ over the right side of the neck. It extended from below the mandible to the level above the cricoid cartilage. The mass splayed the right internal jugular vein and right common carotid artery. The patient was diagnosed with neurogenic tumour and underwent surgical extirpation via a transverse right cervical skin incision. All great vessels were isolated and controlled before the mass was excised. Intraoperatively, the tumour was tracked to the vagal nerve in origin and separated en-bloc from the vagal trunk. The glossopharyngeal, hypoglossal, lingual, accessory nerves were all preserved. The patient was transferred to the intensive care unit after the operation. Laryngeal functions were noted to be normal. During the early postoperative period, the patient developed pneumonia and the neck wound was complicated with an expanding hematoma despite a functioning surgical drain in-situ. The patient underwent a second surgery for wound exploration, hemostasis and hematoma removal after which she was readmitted to the intensive care unit but was not recuperating well. She was kept ventilated and unfortunately succumbed to sepsis secondary to pneumonia one week after surgery. The resected specimen reported findings that were consistent with typical features of schwannoma.

\section{DISCUSSION}

Schwannomas are rare, benign tumours of nerve sheath origin that may originate from any of the cranial, peripheral or autonomic nerves. ${ }^{1}$ Over the last few decades, much literature has been written on the studies on intracranial schwannoma with regards vestibular neuroma. Nonetheless, a majority of schwannomas are non-vestibular and extracranial. Kang et al. reported merely 6 cases of vagal schwannoma identified over the period of 10 years retrospective study. ${ }^{6}$
Extracranial non-vestibular head and neck schwannomas usually present as asymptomatic, slow-growing lateral neck masses that can be palpated along the medial border of the sternocleidomastoid muscle. This renders preoperative diagnosis difficult as many vagal schwannomas do not present with any neurological deficits. ${ }^{5}$ Therefore, possible differentials include metastatic cervical lymphadenopathy, malignant lymphoma, carotid body tumour, branchial cyst, as well as aneurysm. When symptoms are present, hoarseness is the most common symptom for vagal schwannomas. Occasional palpation of the mass may induce paroxysmal coughing. ${ }^{8}$ Horner's syndrome may prevail when the tumour has pressured on the cervical sympathetic chains, or when these chains are themselves the origin of the tumour.

Imaging studies play an important role in the diagnosis of head and neck schwannomas. Regardless of the nerve of origin, schwannomas in general are hypodense in relation to muscle tissue on CT without contrast. With contrast, these lesions may show some degree of enhancement, often peripheral..$^{13}$ More contrast-enhancing lesions should merit differentials of possible vascular lesions. In such instances, angiography or magnetic resonance angiography may be employed to outline the feeding vessels and preoperative embolization could be planned. According to Lin et al. ${ }^{1}$ the site and the way the major neck vessels are displaced could give further clues on the type of schwannoma. Vagal schwannomas typically separate the internal jugular vein and carotid arteries but do not usually widen the carotid bifurcation. On the contrary, sympathetic chain schwannomas mildly splay the carotid bifurcation but do not separate the great vessels. The splaying of the carotid bifurcation is usually more prominent in carotid body tumours. This is called the "lyre sign," which, along with significant contrast enhancement, is rarely associated with schwannoma. ${ }^{14}$

Differentiating amongst the origin of schwannoma can be challenging. In one study, the sympathetic chain was shown to be the most usual site of origin for parapharyngeal schwannoma. ${ }^{4}$ Meanwhile, Maniglia et al. stated that the vagus nerve is the site of origin in approximately $50 \%$ of cases. ${ }^{10}$ The identification of the exact nerve trunk from which the schwannoma arises can be difficult to ascertain. Anatomic evaluation by direct vision intraoperatively is an acceptable way but may not be feasible in all cases. Otherwise, the derivation may be presumed based on the presenting features or postoperative morbidity experienced by the patient. ${ }^{3}$ Nonetheless, adjacent nerves could also be damaged during surgical extirpation, resulting in error of the assumption of origin. Therefore, some diagnoses of the site of origin of schwannoma are definitive; others, presumptive. ${ }^{15}$

Histologically, on hematoxylin and eosin staining, schwannomas show typical clusters of alternating areas of compact hypercellular with spindle shape cells (Antoni A) with areas of loose hypocellular patterns 


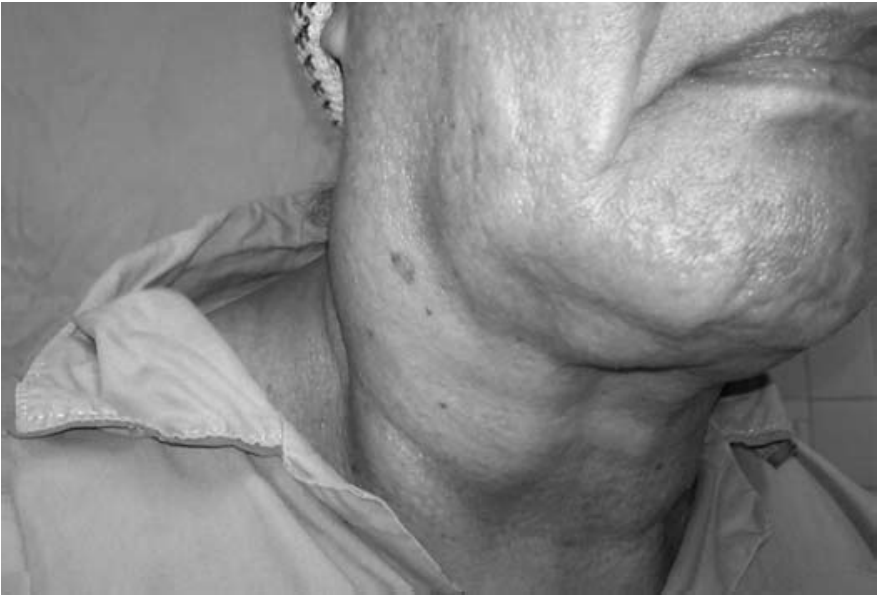

Figure 1. Preoperative appearance of the pulsatile right cervical lesion

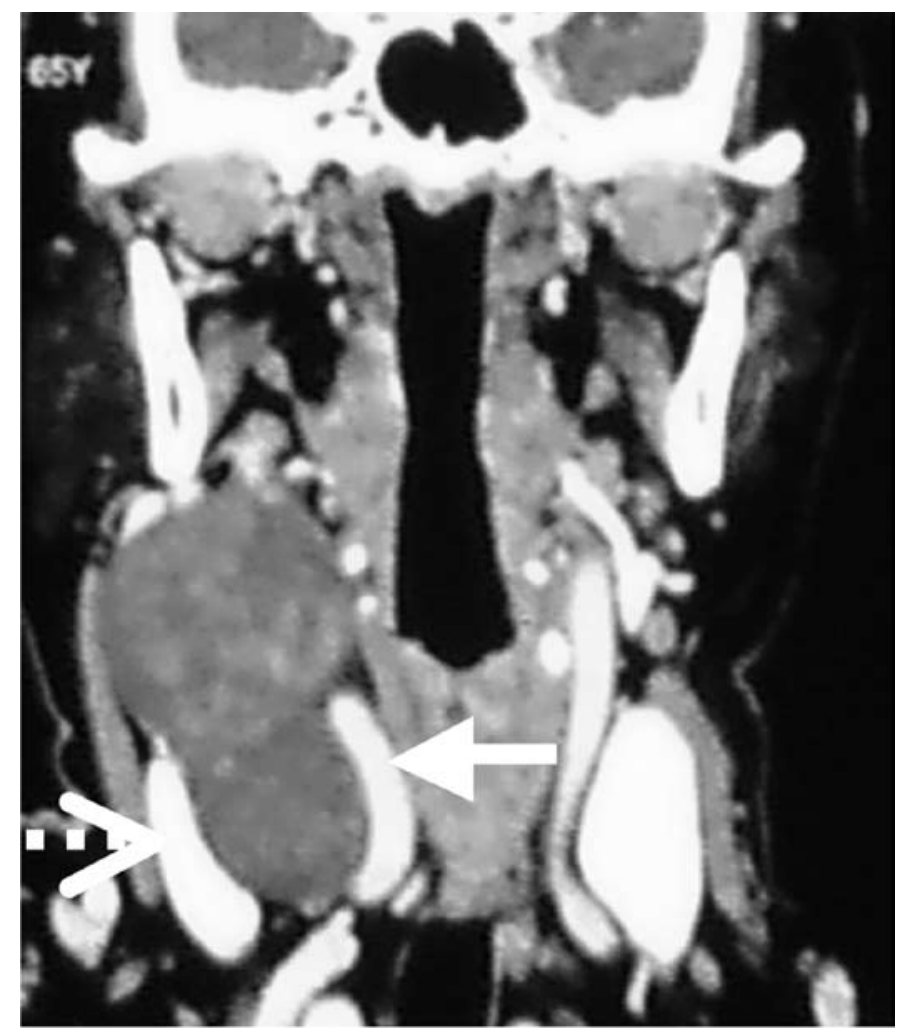

Figure 2. Contrast Computed Tomography of the neck, coronal section: Well-circumscribed dumbbell-shaped heterogenous density in the right parapharyngeal region splaying the right internal jugular vein (dotted arrow) and right carotid artery (solid arrow)

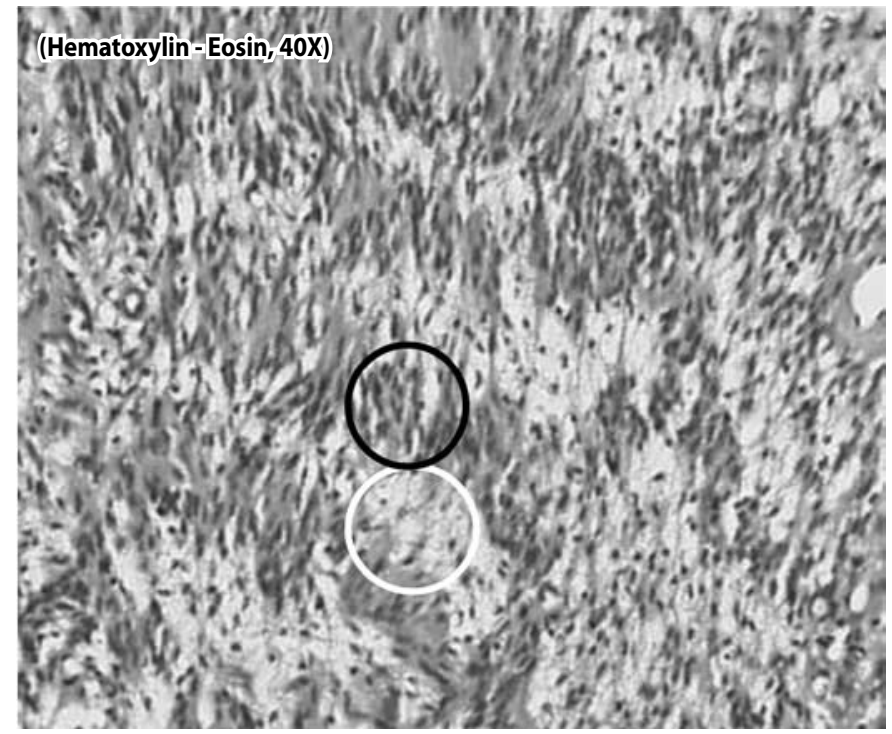

Figure 3. Histopathology (H\&E stain $40 \mathrm{X}$ ) of resected specimen revealed typical benign looking spindle-shape cells arranging themselves in alternating rows of compact hypercellularity and loose hypocellularity, commonly referred to as Antoni Cells A (Black Circle) and Antoni Cells B (White Circle) respectively. Courtesy of Dr Suryati Yusof, Senior Neuropathologist Hospital Kuala Lumpur (Slide No 9076/2009)

(Antoni B). Furthermore, schwannomas also show positivity in S100 protein immunohistochemical studies. Although neurofibromas are of schwann cell origin, they are differentiated from schwannomas by their lack of true capsules and presence of only loose interlacing bands of spindle cells. Moreover, the nerve fibers seen in neurofibromas are found scattered throughout the tumour body whereas those of schwannomas are compressed to one side.

Treatment of vagal schwannomas should encompass complete surgical extirpation with preservation of the neural pathway, as in this case. If the plane of resection is inadequate and preservation is technically difficult, the involved segment of the nerve trunk may be resected with an end-to-end anastomosis using microsurgical techniques. Invariably, this type of procedure will result in vocal cord paralysis or paresis. Therefore, aggressive voice and speech rehabilitation should entail after the surgery.

Morbidities are not uncommon after surgical resection of extracranial nerve sheath tumours. Biswas et al. ${ }^{16}$ reported high complications rates of surgery on benign extracranial nerve sheath tumours. Complications like dysphagia, dysphonia, Horner's syndrome, facial myotonia, hypoglossal palsy, facial palsy and keloid formations have been reported. ${ }^{14-15}$ Nonetheless, neural complications could occur as a result of tumour compression. Therefore there is a need for relevant head and neck neural assessment prior to the surgery so as to correctly identify post-operative neural morbidity. Vascular complications have 
also been reported and are common after intracranial schwannoma resection ${ }^{17}$ but rare for extracranial schwannomas. Rapidly forming hematoma in a neck surgical wound is a dreadful complication and should merit immediate intervention. Tracheostomy may be needed if the airway is compromised. Post surgery atelectasis of the lung is usually the main cause of pneumonia and aggressive chest physiotherapy should be instituted both pre and post operation. Pneumonia may also occur as a result of aspiration secondary to vocal cord palsy possibly due to direct trauma to vagal trunk, traction injury or even compression from hematoma. Because initial laryngeal functions were noted to be normal after the tumour resection in this case, the subsequent wound hematoma could have compressed on the preserved vagal trunk and precipitated aspiration pneumonia.

In conclusion, a patient presenting with solitary mass in the neck should raise the alarm of possible nerve sheath tumour and in the setting of parapharyngeal space involvement, the possibility of arising from cranial nerves, brachial plexus, cervical sympathetic chains or major peripheral nerves. Every effort should be made to preserve the nerve of origin and the patient should be counseled preoperatively on the risk of morbidity especially in the elderly group. Intervention was needed in this case in view of the rapid enlargement of the cervical swelling that could signify malignant transformation or anticipated compressive symptoms. Surgical extirpation is still the treatment of choice for nerve sheath tumours and tumour recurrence is uncommon. Nonetheless, in view of the possible surgical morbidities, close follow up and aggressive rehabilitation should be instituted following surgical treatment.
REFERENCES

1. Lin C, Wang C, Liu S, Wang C, Chen W. Cervical sympathetic chain schwannoma. J Formos Med Assoc. 2007; 106(11): 956-960.

2. Al-Ghamdi S, Black MJ, Lafond G. Extracranial head and neck schwannomas. J Otolaryngol 1992 Jun; 21(3): 186-8.

3. Daly JF, Roesler HK, Neurilemmoma of the cervical sympathetic chain. Arch Otolaryngol 1963 Mar; 77:262-6.

4. Fluur E. "Parapharyngeal" neurogenic tumors. J Laryngol Otol. 1965; 79:796-805.

5. Toriumi DM, Anyah RA, Murad T, Sisson GA. Extracranial neurogenic tumors of the head and neck. Otolaryngol Clin North Am 1986 Aug; 19(3):609-17.

6. Kang GCW, Soo KC, Lim DTH. Extracranial non-vestibular head and neck schwannomas: a tenyear experience. Ann Acad Med Singapore. 2007 Apr; 36(4):233-240.

7. Chiofalo MG, Longo F, Marone U, Franco R, Petrillo A, Pezzullo L. Cervical vagal schwannoma: A case report. Acta Otorhinolaryngol Ital. 2009 Feb; 29(1):33-35.

8. Ford LC, Cruz RM, Rumore GJ, Klein J. Cervical cystic schwannoma of the vagus nerve: diagnostic and surgical challenge. J Otolaryngol. $2003 \mathrm{Feb} ; 32(1): 61-63$.

9. Takimoto T, Katoh H, Umeda R. Parapharyngeal schwannoma of the cervical symphathetic chain in a child. Int J Pediatr Otorhinolaryngol. 1989 Sep; 18(1): 53-58.

10. Maniglia AJ, Chandler JR, Goodwin WJ, Parker JC Jr.. Schwannomas of the parapharyngeal space and mid jugular foramen. Laryngoscope. 1979; 89(9):1405-14.

11. Agrawal A, Pandit L, Bhandary S, Makannavar JH, Srikrishna U. Glossopharyngeal schwannoma: diagnostic and therapeutic aspects. Singapore Med J. 2007 Jul; 48(7): e181-5.

12. Wilson JA, McLaren K, McIntyre MA, von Haacke NP, Maran AGD. Nerve-sheath tumours of the head and neck. Ear Nose Throat J. 1988; 67:103-110.

13. Furukawa M, Furukawa MK, Katoh K, Tsukuda M. Differentiation between schwannoma of the vagus nerve and schwannoma of the cervical sympathetic chain by imaging diagnosis. Laryngoscope 1996; 106:1548-52.

14. Olsen KD. Tumors and surgery of the parapharyngeal space. Laryngoscope 1994 May; 104(5 Pt 2 Suppl 63):1-28.

15. Sheridan MF, Yim DW. Cervical sympathetic schwannoma: a case report and review of the English literature. Otolaryngol Head Neck Surg. 1997 Dec; 117(6):S206-210.

16. Biswas D, Marnane C, Mal R, Baldwin D. Benign extracranial nerve sheath tumors of the skull base: postoperative morbidity and management. Skull Base. 2008 Mar;18(2):99-106.

17. Sade B, Mohr G, Dufour JJ. Vascular complications of vestibular schwannoma surgery: a comparison of the suboccipital retrosigmoid and translabyrinthine approaches. J Neurosurg. 2006 Aug; 105(2):200-204. 